\title{
RELAÇÃO ENTRE DESMAME PRECOCE E ALERGIAS ALIMENTARES
}

\author{
RELATIONSHIP BETWEEN EARLY WEANING AND FOOD ALLERGIES
}

\section{Dayane Kanarski Bernardino JOSÉ1'; Jéssica Antunes VITIATO'1 Karina HASS'; Tauani Caroline Santos FRANÇA ${ }^{1}$; Mariana Abe Vicente CAVAGNARI ${ }^{2}$}

\author{
1 - Acadêmica da Universidade Estadual do Centro-Oeste \\ 2 - Nutricionista, Docente do curso de graduação em Nutrição da Universidade Estadual do Centro-Oeste. \\ Autor para correspondência: dayane_kanarski@hotmail.com
}

\section{RESUMO:}

A amamentação desempenha um papel importante na saúde dos neonatos, sendo o leite materno o alimento que fornece os nutrientes que a criança necessita para um adequado crescimento e desenvolvimento e oferece efeitos protetores essenciais para a redução de alergias alimentares, que está relacionada com interferências nutricionais nessa fase da vida. O presente estudo teve como objetivo investigar na literatura científica as relações entre a hipersensibilidade alimentar com o desmame precoce, verificando a associação entre esses dois conceitos abordando as principais causas que levam ao desenvolvimento de alergias alimentares. Foi realizada uma pesquisa de revisão de literatura nas bases de dados SCIELO, LILACS, SCIENCE DIRECT, PUBMED e MEDLINE, com ênfase em artigos dos últimos 15 anos, referente à relação do desmame precoce e reações alergênicas. Por meio desta revisão, verificou-se que muitas são as causas que levam à interrupção do aleitamento materno, como a ausência de informações sobre a importância do aleitamento materno pelas mães, introdução precoce de alimentos e o retorno das mães ao trabalho. Observa-se que os lactentes, principalmente, nos primeiros meses de vida apresentam o sistema imunológico e gastrointestinal imaturo, estando mais susceptíveis a absorção de macromoléculas e ao desenvolvimento de reações de hipersensibilidade, que podem desencadear alergias alimentares.

Palavras-chave: amamentação; nutrição; sistema imunológico.

\section{ABSTRACT:}

Breastfeeding plays an important role in the health of newborns, with breastmilk being the food that provides the nutrients that the child needs for adequate growth and development and provides essential protective effects for the reduction of food allergies, which is related to nutritional interferences in this Stage of life. The present study aimed to investigate in the scientific literature the relationships between food hypersensitivity and early weaning, verifying the association between these two concepts, addressing the main causes that lead to the development of food allergies. A literature review was conducted in the databases SCIELO, LILACS, SCIENCE DIRECT, PUBMED and MEDLINE, with emphasis on articles from the last 15 years, regarding the relationship of early weaning and allergenic reactions. Through this review, it was verified that there are many causes that lead to the interruption of breastfeeding, such as the lack of information about the importance of breastfeeding by mothers, the early introduction of food and the return of mothers to work. It is observed that infants, especially in the first months of life, have the immature and immature gastrointestinal 
system, being more susceptible to the absorption of macromolecules and the development of hypersensitivity reactions, which can trigger food allergies.

Keywords: breast-feeding; nutrition; immune system

\section{INTRODUÇÃO}

O leite materno é de suma importância para os neonatos, sendo a forma mais completa de alimentação antes da introdução da alimentação complementar, pois contêm características nutricionais e imunológicas, que suprem as necessidades alimentares da criança, proporcionando um adequado crescimento e desenvolvimento, estabelecendo o vínculo de mãe para filho, além de ter implicações na saúde física e psíquica das nutrizes (Ministério da Saúde, 2009). É também considerado um alimento com efeito protetor contra doenças crônicas não-transmissíveis, infecções gastrointestinais e respiratórias e alergias e intolerâncias alimentares (PEREIRA, 2010; TOMA, 2008).

O aleitamento materno é considerado exclusivo quando este é ofertado sem a presença de outros alimentos na dieta dos lactentes e ofertado sobre livre demanda. A recomendação da Organização Mundial da Saúde (OMS) é que as crianças recebam leite materno por dois anos ou mais, sendo exclusivo nos primeiros seis meses e após os seis meses é necessário à introdução de alimentos complementares para suprir as necessidades que aumentam conforme seu desenvolvimento. (OMS, 2002; OLIVEIRA, 2015).

O desmame precoce é caracterizado pela ausência de amamentação ou sua interrupção precoce e a introdução de outros tipos de alimentos na dieta da criança antes dos seis meses de idade (BATISTA, 2009). A substituição do leite materno por outros alimentos pode desencadear problemas de saúde e a ocorrência de doenças, pois os lactentes principalmente nos primeiros meses de vida apresentam o sistema imunológico e gastrointestinal imaturo, estando mais susceptíveis a absorção de macromoléculas e ao desenvolvimento de reações de hipersensibilidade podendo desencadear alergias alimentares (FERREIRA e SEIDMAN, 2007).

Com base nos dados da Pesquisa Nacional de Demografia e Saúde da Criança e da Mulher (PNDS) realizada em 2006, é possível observar que no Brasil a duração média de aleitamento materno exclusivo é de apenas 1,4 meses, 35\% das crianças recebem amamentação exclusiva durante os primeiros quatro meses de vida e já no primeiro mês de vida $25 \%$ das crianças entre 3 e 6 meses já consumem comida salgada e frutas (BRASIL, 2009; MONTEIRO et al, 2011).

Estima-se que as alergias alimentares vêm apresentando um aumento nas ultimas 
décadas, em que a prevalência é de $6 \%$ a $8 \%$, afetando principalmente crianças menores de três anos de idade em decorrência a exposição cada vez mais precoce e inadequada de alimentos complementares á dieta de crianças nessa faixa etária. A substituição do leite materno pelo leite de vaca antes dos seis meses de vida aumenta a probabilidade do desenvolvimento de alergias alimentares, mas quaisquer alimentos oferecidos aos lactentes precocemente podem desencadear reação alérgica, sendo os mais comuns o leite de vaca, soja, ovos, amendoim, castanhas, camarão e peixe (ASBAI, 2006; PEREIRA, 2008).

O objetivo do presente estudo foi investigar na literatura científica as relações entre a hipersensibilidade alimentar com o desmame precoce, verificando a associação entre esses dois conceitos abordando as principais causas que levam ao desenvolvimento de alergias alimentares em neonatos.

\section{MATERIAL E MÉTODOS}

O presente estudo trata-se de uma revisão integrativa cuja metodologia é baseada na leitura e análise de estudos de caráter científico relacionados à amamentação e alergias alimentares.

A revisão integrativa teve como primeira etapa percorrida a escolha da temática e posterior consulta de estudos na área. Os descritores utilizados na busca foram "Aleitamento materno", "Desmame precoce”, "Reação alérgica”. A bibliografia consultada incluiu resumos disponíveis e indexados nas bases de dados: SCIELO, LILACS, SCIENCE DIRECT, PUBMED e MEDLINE. Os critérios de inclusão adotados pelo presente estudo foram artigos que apresentassem como objetivo demonstrar a importância do aleitamento materno exclusivo ou que estivessem relacionados a alergias alimentares. Os critérios de exclusão foram artigos publicados há mais de 15 anos ou que não apresentassem resultados relevantes e publicações de revisões literárias.

Foram analisados seis artigos para o desenvolvimento do processo de escrita do presente artigo. A análise dos estudos para posterior discussão considerou a leitura dos resumos dos trabalhos selecionados para descrição de forma clara e objetiva os resultados encontrados em cada estudo.

\section{RESULTADOS}

Foram identificados treze estudos que tratavam do tema abordado, que após serem selecionados foram lidos na íntegra. Após a análise, apenas seis foram incluídos por estarem de acordo com os critérios de inclusão e exclusão. Foi realizado panorama geral 
Quadro 1. EVIDENCIAS CIENTÍFICAS SOBRE A RELAÇÃO ENTRE DESMAME PRECOCE E ALERGIAS ALIMENTARES.

\begin{tabular}{|c|c|c|c|}
\hline Autor/Ano & Amostra & Objetivo & Conclusões \\
\hline $\begin{array}{l}\text { Calza - Brasília, } \\
\text { Brasil (2012) }\end{array}$ & $\begin{array}{l}152 \text { pais de } 280 \\
\text { crianças } \\
<5 \text { anos }\end{array}$ & $\begin{array}{l}\text { Analisar a relação do } \\
\text { desmame precoce com a } \\
\text { prevalência das alergias e } \\
\text { intolerâncias alimentares. }\end{array}$ & $\begin{array}{c}\text { Há evidências para se } \\
\text { afirmar que crianças com } \\
\text { tempo de aleitamento menor } \\
\text { tenham maior propensão a } \\
\text { alergias. }\end{array}$ \\
\hline $\begin{array}{l}\text { Machado et al - } \\
\text { Rio de Janeiro, } \\
\text { Brasil (2014) }\end{array}$ & $\begin{array}{l}170 \text { puérperas } \\
\text { com idade } \\
\text { média de } 26,5 \\
\pm 5,8 \text { anos. }\end{array}$ & $\begin{array}{l}\text { Analisar a intenção de } \\
\text { puérperas de amamentar e } \\
\text { introdução de alimentos } \\
\text { complementares no primeiro } \\
\text { ano de vida. }\end{array}$ & $\begin{array}{l}\text { O tempo de amamentação } \\
\text { exclusiva foi aquém do } \\
\text { preconizado pela OMS e a } \\
\text { alimentação complementar } \\
\text { mostrou-se equivocada. }\end{array}$ \\
\hline $\begin{array}{l}\text { Saliba et al-São } \\
\text { Paulo, Brasil } \\
\text { (2008) }\end{array}$ & $\begin{array}{c}100 \text { mães de } \\
\text { crianças com } \\
\text { até } 12 \text { meses de } \\
\text { idade }\end{array}$ & $\begin{array}{l}\text { Avaliar a prevalência do } \\
\text { aleitamento materno e } \\
\text { associação variáveis } \\
\text { sociodemográficas e } \\
\text { conhecimento das mães } \\
\text { sobre a relação da } \\
\text { amamentação. }\end{array}$ & $\begin{array}{l}\text { Foram baixas as taxas de } \\
\text { aleitamento exclusivo, e como } \\
\text { fatores determinantes } \\
\text { destacaram-se o uso de } \\
\text { mamadeiras e chupetas } \\
\text { associado ao desmame. }\end{array}$ \\
\hline $\begin{array}{l}\text { Schincaglia et al } \\
\text { - Goiânia, Brasil } \\
\text { (2015) }\end{array}$ & $\begin{array}{c}362 \text { recém- } \\
\text { nascidos de } \\
\text { ambos os sexos }\end{array}$ & $\begin{array}{c}\text { Analisar as práticas } \\
\text { alimentares e fatores } \\
\text { associados à introdução } \\
\text { precoce da alimentação } \\
\text { complementar entre } \\
\text { crianças menores de } \\
\text { seis meses nascidas em } \\
\text { maternidade na região } \\
\text { noroeste de Goiânia-GO, } \\
\text { Brasil. }\end{array}$ & $\begin{array}{l}\text { A introdução precoce da } \\
\text { alimentação complementar } \\
\text { influenciou negativamente na } \\
\text { duração do aleitamento } \\
\text { materno exclusivo, bem menor } \\
\text { que a recomendada pela OMS } \\
\text { e Ministério de Saúde. }\end{array}$ \\
\hline $\begin{array}{l}\text { Brustolin et al, } \\
\text { Guarapuava, } \\
\text { Brasil (2009) }\end{array}$ & $\begin{array}{c}102 \text { mães de } \\
\text { crianças } \\
\text { menores de seis } \\
\text { meses da região } \\
\text { urbana do } \\
\text { município de } \\
\text { Guarapuava }\end{array}$ & $\begin{array}{c}\text { Verificação da } \\
\text { introdução precoce da } \\
\text { alimentação } \\
\text { complementar, bem } \\
\text { como o mês e os } \\
\text { motivos maternos, em } \\
\text { crianças menores de } \\
\text { seis meses de vida na } \\
\text { região urbana do } \\
\text { município de } \\
\text { Guarapuava PR. }\end{array}$ & $\begin{array}{l}\text { Grande parte das mães } \\
\text { ainda introduz alimentos } \\
\text { complementares antes dos } \\
\text { seis meses de vida da } \\
\text { criança. A introdução de } \\
\text { alimentos é altamente } \\
\text { influenciando por hábitos } \\
\text { sócio-culturais. }\end{array}$ \\
\hline $\begin{array}{l}\text { Graciete et al - } \\
\text { Rio de Janeiro, } \\
\text { Brasil (2004) }\end{array}$ & $\begin{array}{l}\text { Questionário foi } \\
\text { aplicado às } \\
2.323 \text { mães das } \\
\text { crianças }\end{array}$ & $\begin{array}{l}\text { Descrever os hábitos } \\
\text { alimentares de crianças } \\
\text { amamentadas e não- } \\
\text { amamentadas através } \\
\text { da análise dos alimentos } \\
\text { consumidos pelas } \\
\text { crianças menores de } 1 \\
\text { ano de idade }\end{array}$ & $\begin{array}{l}\text { As crianças amamentadas, } \\
\text { quando comparadas com as } \\
\text { não-amamentadas, } \\
\text { apresentaram menores chances } \\
\text { de desenvolvimento de alergias } \\
\text { alergias alimentares }\end{array}$ \\
\hline
\end{tabular}




\section{DISCUSSÃO}

No estudo realizado por Calza (2012), com 152 pais de 280 crianças $<5$ anos, o retorno ao trabalho e a opção materna foram as principais causas que influenciaram a realizar o desmame precoce, concordando com o estudo realizado por Brustolin (2009) com 102 mães de crianças menores de 6 meses, em que a volta ao trabalho e a opção materna também influenciaram as mães a pararem de amamentar, relatando que existem vários prejuízos com esta prática, dentre elas, uma maior vulnerabilidade ao aparecimento de alergias.

Para validar esta informação, Calza (2012) analisou a relação do desmame precoce com a prevalência das alergias e intolerâncias alimentares, prevalecendo à alergia do leite de vaca e a intolerância a lactose. Como muitas mães acreditam que o leite de vaca pode substituir o leite materno ou que não há problemas em oferecê-lo antes dos 6 meses, pode haver uma relação entre a prevalência de alergias ao leite de vaca com a introdução precoce desse alimento, pois há várias evidências de que além de não substituir o leite materno ainda pode causar várias consequências negativas, como o desenvolvimento de alergias alimentares.

O estudo de Graciete (2004) também explica o grande número de crianças que desenvolvem intolerância sendo principalmente ao leite de vaca, não só pelo alto poder antigênico, como também pela precocidade do seu uso, o leite de vaca é responsável por $20 \%$ das alergias alimentares. Em seu estudo foram aplicados questionários com as mães das crianças menores de 1 ano correspondendo a 2.319 crianças, a fim de avaliar os hábitos alimentares de crianças amamentadas e não-amamentadas. Nas crianças com idade igual ou inferior a 4 meses e não-amamentadas, foi observada uma chance 8,2 e 6,7 vezes maior de a criança ser alimentada, com papas de legumes, água, chás, sucos e papa de frutas, outro fator que além de influenciar no desmame precoce pode ainda causar intolerância alimentar.

No estudo realizado por Schincaglia (2015), a maioria das mães analisadas tinham idade entre 20 e 35 anos, apresentavam baixa escolaridade e não trabalhava fora do lar. Entre as crianças, $59,1 \%$ fizeram o uso de chupeta e $62,5 \%$ de mamadeira, nos primeiros seis meses de vida, sendo que tais fatores podem ter influenciado a ocorrência de desmame, concordando com os estudos realizados por Saliba (2014) e Venancio (2002), em que abordaram fatores como escolaridade materna e a maternidade precoce, nos quais identificaram que o trabalho informal e o desemprego tiveram maior influência no desmame 
precoce em crianças menores de quatro meses.

SCHINCAGLIA (2015) observou que houve baixa prevalência ao aleitamento materno exclusivo. Segundo os autores, a introdução de alimentos antes dos seis meses de vida ocasiona prejuízos à saúde do bebê, dentre eles, um maior risco para as alergias em função da imaturidade fisiológica, o que pode ser comprovado pelo estudo realizado por Calza (2012), em que 7\% das crianças que foram desmamadas precocemente, apresentou alergias e intolerâncias alimentares, o que sugere alguma relação entre esses fatores.

O uso de mamadeiras e chupetas já haviam sido associados ao desmame precoce por Bucciniet (2014). Segundo esses autores, esses utensílios causam desmame precoce devido à substituição do "peito" da mãe pelo bico artificial diminuindo o estímulo ao mamilo e, portanto, reduzindo a produção láctea. Desta forma, a presença de hábitos de sucção foi considerada um fator protetor da amamentação. No estudo de Machado (2014), as puérperas avaliadas tiveram informações sobre aleitamento materno exclusivo durante as consultas pré natais, no qual obteve-se resultados satisfatórios em relação ao tempo médio pretendido de 5,5 meses de aleitamento exclusivo pelas mães, ou seja, próximo ao tempo preconizado pela OMS. Entretanto, estes resultados diferem significativamente aos encontrados na literatura. Em estudo realizado na cidade de Maringá (BERCINI, 2007) constatou-se que apenas $25 \%$ das crianças com seis meses de idade estavam sendo amamentadas exclusivamente. Bem como no estudo de Saliba (2008) citado anteriormente apenas $22 \%$ estavam recebendo aleitamento exclusivo. Morgado et al., na cidade do Rio de Janeiro, apenas $16 \%$ dos bebês eram alimentadas exclusivamente com leite materno. Esta diferença nos resultados pode ser atribuída ao fato de que o estudo de Machado se trata de uma pretensão e os demais ao que de fato ocorreu.

Apesar da divulgação da informação sobre a importância do aleitamento materno exclusivo. O conhecimento dessa relação não é de domínio popular nem menos da metade das mães demonstraram saber a influência que o aleitamento pode exercer na saúde de seus filhos. A comparação dos resultados dessas pesquisas demonstra que as deficiências de conhecimento são semelhantes e devem ser partilhadas para que ações abrangentes sejam instituídas como medidas de combate ao desmame precoce no país.

As evidências que relacionam o desmame precoce com o surgimento de alergias alimentares são muitas, porém, há poucos estudos que comprovem o fato, com isso, podemos constatar que há uma necessidade de maiores estudos nessa área, visando a implantação de políticas públicas para redução de desenvolvimento de patologias associadas ao desmame precoce. 


\section{CONCLUSÃO}

A imaturidade do sistema imunológico dos neonatos está diretamente relacionada à sensibilização alérgica, correlacionando o aparecimento de alergias em crianças que foram privadas do aleitamento materno exclusivo, o que pode também trazer outras consequências à saúde como a exposição a agentes infecciosos que podem causas reações alérgicas.

Considerando os benefícios já descritos anteriormente na literatura sobre a prevenção de alergias alimentares se mantido o aleitamento materno exclusivo até os 6 meses de idade. Com base nos resultados apresentados, sugere-se estudos que abordem essa temática e que orientem novas estratégias de conscientização sobre a sua importância, afim de minimizar o risco de desenvolvimento de doenças ou alergias.

\section{REFERÊNCIAS}

Associação Brasileira de Alergia e Imunopatologia - ASBAI. Alergia alimentar- 2006. Disponível em: http://www.asbai.org.br/secao.asp?s=81\&id=306. Acessado em 06 de set. 2016

AUDI, C.A.F., et al. Alimentos complementares e fatores associados ao aleitamento materno e ao aleitamento materno exclusivo em lactentes até 12 meses de vida em Itapira, São Paulo, 1999. Rev Bras Saúde Matern Infant.v. 3, p. 85-93, 2003

BATISTA, G. S.; FILHUSI, A. M. F.; HAACK, A. Alergia alimentar e desmame precoce: uma revisão do ponto de vista nutricional. Com. Ciências Saúde, v. 20, n. 4, p. 351-360, 2009.

BERCINI, L.O., et al. Alimentação da criança no primeiro ano de vida, em Maringá, PR. CiencCuidSaude; v.6, n.2, p.404-410, 2007.

BRASIL. Ministério da Saúde, Pesquisa Nacional de Demografia e Saúde da Criança e da Mulher - PNDS 2006: dimensões do processo reprodutivo e da saúde da criança, Centro Brasileiro de Análise e Planejamento.2009. 
menores de seis meses de vida. 2009. 16 p. Trabalho de Conclusão de Curso. (Graduação em Nutrição). Universidade Estadual do Centro Oeste, Guarapuava, 2009.

BUCCINI, G. S.; BENÍCIO, M. H. VENANCIO, I. Determinantes do uso de chupeta e mamadeira.Rev Saúde Pública, v.48, n.4, p. 571-582, 2014.

CALZA, G. F. Relação entre Desmame Precoce e Alergias Alimentares em Crianças Matriculadas em Duas Instituições Filantrópicas de Brasília. Centro Universitário de Brasília - UNICEUB Faculdade de Ciências da Educação e Saúde - FACES, Brasília, 2012.

FERREIRA, C. T. et al.Alergia Alimentar: atualização prática do ponto de vista gastroenterológico, Jornal de Pediatr., v. 83, n.1, p.7-20, 2007.

GRACIETE, O. V.; LUCIANA R. S.; TATIANA, O. V. Hábitos alimentares de crianças menores de 1 ano amamentadas e não-amamentadas. Jornal de Pediatria, Rio de Janeiro. v. 80, n.5, 2004.

MACHADO, A. K. F.; et al. Intenção de amamentar e de introdução de alimentação complementar de puérperas de um Hospital-Escola do sul do Brasil. Rio de Janeiro,Ciênc. saúde coletiva v.19 n.7, 2014.

Ministério da Saúde, Secretaria de Atenção à Saúde, Departamento de Ações Programáticas e Estratégicas. II Pesquisa de Prevalência de Aleitamento Materno nas Capitais Brasileiras e Distrito Federal. 2009. 1ํedição.

Ministério da Saúde. Secretaria de Atenção à Saúde. Departamento de Atenção Básica. Saúde da Criança: nutrição infantil: aleitamento materno e alimentação complementar. Brasília: Editora MS, 2009.

MONTEIRO S.C. et al. O Aleitamento Materno Enquanto uma Prática Construída: Reflexões acerca da evolução histórica da amamentação e desmame precoce no Brasil. Investigaç. e Educ. em Enferm. v.29, n.2., p.315-321, 2011.

MORGADO C. M.C et al. Rede e apoio social e práticas alimentares de crianças no 
quarto mês de vida. CienSaudColet,v.18, n.2, p.367-376, 2013.

OLIVEIRA, R. C; et al. Uso de chupeta e desmame precoce: Uma revisão de literatura. Ver. Saúde. Com. v.11 n.22. p. 183-192, 2015.

PEREIRA, A. C. S et al. Alergia Alimentar: sistema imunológico e principais alimentos envolvidos. Semina: Ciências Biológicas e da Saúde, Londrina, v.29, n.2, p.189 - 200, jul./dez.2008.

SALIBA, N. A.Frequência e variáveis associadas ao aleitamento materno em crianças de até 12 meses de idade, em Araçatuba, Estado de São Paulo, Brasil. Rev Bras Saúde Mater Infant. v.8, n.4, p.481-90, 2008.

SCHINCAGLIA, R.M. et al. Práticas alimentares e fatores associados à introdução precoce da alimentação complementar entre crianças menores de seis meses na região noroeste de Goiânia. Epidemiol. Serv. Saúde vol.24 n.3 Brasília, 2015.

TOMA, T.S; et al. Benefícios da Amamentação para a Saúde da Mulher e da Criança: um ensaio sobre as evidências, Caderno de Saúde Pública, Rio de Janeiro, v.24, n.2, p.235-246, 2008.

VIEIRA, G. et al.Fatores associados ao aleitamento materno e desmame em Feira de Santana, Bahia. Rev. Bras Saúde Matern Inf. v.4, p.143-50, 2004. 